\title{
Label-Free Identification of Early Stages of Breast Ductal Carcinoma via Multiphoton Microscopy
}

\author{
Zhong Chen, ${ }^{1}$ Wenhui Guo, ${ }^{2}$ Deyong Kang, ${ }^{3}$ Shu Wang, ${ }^{4}$ Liqin Zheng, ${ }^{1}$ Gangqin Xi $\mathbb{D},{ }^{1}$ \\ Yuane Lian, ${ }^{3}$ Chuan Wang $\left({ }^{2},{ }^{2}\right.$ and Jianxin Chen $\mathbb{D}^{1}$ \\ ${ }^{1}$ Key Laboratory of OptoElectronic Science and Technology for Medicine of Ministry of Education, Fujian Provincial Key Laboratory \\ of Photonics Technology, Fujian Normal University, Fuzhou 350007, China \\ ${ }^{2}$ Department of Breast Surgery, Fujian Medical University Union Hospital, Fuzhou 350001, China \\ ${ }^{3}$ Department of Pathology, Fujian Medical University Union Hospital, Fuzhou 350001, China \\ ${ }^{4}$ College of Mechanical Engineering and Automation, Fuzhou University, Fuzhou 350116, China
}

Correspondence should be addressed to Chuan Wang; chuanwang1968@hotmail.com and Jianxin Chen; chenjianxin@fjnu.edu.cn Received 15 October 2019; Accepted 3 March 2020; Published 2 April 2020

Academic Editor: Michela Relucenti

Copyright (C) 2020 Zhong Chen et al. This is an open access article distributed under the Creative Commons Attribution License, which permits unrestricted use, distribution, and reproduction in any medium, provided the original work is properly cited.

Breast cancer can be cured by early diagnosis. Appropriate and effective clinical treatment benefits from accurate pathological diagnosis. However, due to the lack of effective screening and diagnostic imaging methods, early stages of breast cancer often progress to malignant breast cancer. In this study, multiphoton microscopy (MPM) via two-photon excited fluorescence combined with second-harmonic generation was used for identifying the early stages of breast ductal carcinoma. The results showed differences in both cytological features and collagen distribution among normal breast tissue, atypical ductal hyperplasia, low-grade ductal carcinoma in situ, and high-grade ductal carcinoma in situ with microinvasion. Furthermore, three features extracted from the MPM images were used to describe differences in cytological features, collagen density, and basement membrane circumference in the early stages of breast ductal carcinoma. They revealed that MPM has the ability to identify early stages of breast ductal carcinoma label-free, which would contribute to the early diagnosis and treatment of breast cancer. This study may provide the groundwork for the further application of MPM in the clinic.

\section{Introduction}

Breast cancer is the most frequent cancer in women and the second most common cancer causing female death, with estimated 266,120 new cases and 40,920 deaths in 2018 in the USA [1]. However, $70 \%-75 \%$ of breast cancers are associated with breast ductal lesions [2]. Atypical ductal hyperplasia $(\mathrm{ADH})$ is an indirect precursor of invasive ductal carcinoma, and ductal carcinoma in situ (DCIS) is an immediate precursor of invasive ductal carcinoma [3-6]. Ductal carcinoma in situ with microinvasion (DCIS-MI) is a prestage of breast ductal carcinoma $[7,8]$. If these early lesions are detected in time, diagnosed accurately, and treated early, patients will have a good prognosis. DCIS is a group of highly heterogeneous neoplastic intraductal lesions that classify into lowgrade DCIS, intermediate-grade DCIS, and high-grade DCIS, primarily based on the nuclear grade or necrosis [9]. Atypical hyperplasia of $\mathrm{ADH}$ is similar to proliferating cells of lowgrade DCIS, but the extent of ADH is small, with less than two lumens completely involved, or lesions less than $2 \mathrm{~mm}$ $[10,11]$. DCIS-MI is characterized by ductal carcinoma in situ, while some cancer cells break through the basement membrane of the duct system into adjacent stromal tissue with no foci more than $1 \mathrm{~mm}$ of the maximum diameter [8]. DCISMI often occurs in high-grade DCIS with a wide range of lesions but can also be seen in any grade of DCIS $[7,8]$. However, due to the lack of accurate and rapid screening as well as diagnostic imaging methods, these lesions often progress to malignant ductal carcinoma and even distant metastases.

Currently, pathologists identify these early stages of breast ductal carcinoma through hematoxylin and eosin(H\&E-) stained sections of needle biopsy specimens or 
postoperative specimens. Although ADH and DCIS are easily identified by H\&E sections, the identification of DCISMI is difficult. Pathologists identify acute microinvasive foci and other suspected lesions by using immunostaining markers to label myoepithelial cells around the tumor and seeing the presence of myoepithelial cell layers. However, when reslicing to detect immunostaining markers of infiltrates, microinvasive lesions may no longer appear on the sections. In addition, histopathological examination of $\mathrm{H} \& \mathrm{E}$ sections has several other shortcomings, including timeconsuming and labor-intensive pathological processes and potential risk bias. Therefore, there is an urgent need for developing fast and efficient optical imaging methods that can be used for screening and diagnosing these early lesions.

Multiphoton microscopy (MPM) via two-photon excited fluorescence (TPEF) combined with second-harmonic generation (SHG) is a promising, label-free, and high-resolution imaging technique for cancer research and diagnosis [1215]. There are abundant autofluorescent substances in breast tissue, such as elastic fibers, $\mathrm{NAD}(\mathrm{P}) \mathrm{H}$, and FAD, which can generate TPEF signals $[16,17]$; asymmetric collagen such as type I collagen, type II collagen, type III collagen, and type V collagen is more likely to produce SHG signals [18]. Particularly, SHG imaging visualizes the microstructure of collagen fibers and elastin fibers in the extracellular matrix by detecting tissue intrinsic fluorescence [19]. On the other hand, TPEF imaging can directly observe changes in cellular morphology and organization [12]. High-contrast SHG and TPEF overlay images highlight collagen reorganization in the tumor-stromal interface, which is associated with cancer progression. Hence, in this study, we sought to examine whether MPM imaging can identify early stages of breast ductal carcinoma.

\section{Materials and Methods}

2.1. Sample Preparations. In this work, 16 cases of paraffinembedded breast cancer samples, including 5 normal breast tissues, 3 ADH, 5 low-grade DCIS, and 3 high-grade DCISMI, were obtained from the pathological department of Fujian Union Hospital. The patients enrolled in the study were diagnosed at 21-75 years old. The Institutional Review Committee of Fujian Union Hospital (Fuzhou, China) approved of this study, and patients signed informed consent prior to participating in this study. Five consecutive sections of $5 \mu \mathrm{m}$ thickness were used in this study, and after paraffin section dewaxing, the middle slice was stained with $\mathrm{H} \& \mathrm{E}$ to further determine experimental results; the other four sections were used for multiphoton imaging.

2.2. MPM System. The MPM system used in this work has been described in detail before [20]. Briefly, a commercial laser scanning microscope (Zeiss LSM 880 META, Jena, Germany) equipped with an external automode-locked Ti:sapphire laser (140 fs, $80 \mathrm{MHz}$ ) was used for all MPM imaging. Unstained samples were excited by $810 \mathrm{~nm}$ light from the tunable Ti:sapphire laser (Chameleon Ultra, Coherent, Inc., Santa Clara, CA, USA). A 20x Plan-Apochromat objective (NA $=0.8$, Zeiss, Jena, Germany) was used for get- ting backscattered fluorescence, which was separated by passing through a grating onto 32-channel GaAsP and PMT array detectors to obtain a TPEF signal and SHG signal, respectively. MPM images were obtained from two separate channels simultaneously: (1) the TPEF channel gathered $430 \mathrm{~nm}-695 \mathrm{~nm}$ fluorescence with the 32-channel GaAsP detectors (color-coded red); (2) the SHG channel gathered $389 \mathrm{~nm}-419 \mathrm{~nm}$ fluorescence with a PMT detector (colorcoded green). The LSM software automatically spliced out an image array with a resolution of $1024 \times 1024$ pixels per frame to form a large-area image. All MPM images had a 12-bit pixel depth; the images were obtained at $0.77 \mu \mathrm{s} /$ pixel. The maximum scanning speed in the bidirectional scanning mode was 1.6 frames/sec $(1024 \times 1024$ pixels $)$.

2.3. Histologic Analysis. H\&E-stained digital images (40x) were obtained from an optical microscope (Eclipse Ci-L, Nikon Instruments, Japan) with CCD (DS-Fi2, Nikon) and confirmed by two experienced pathologists (Deyong Kang and Yuane Lian), reducing subjective observational errors. Then, two researchers (Liqin Zheng and Zhong Chen) confirmed the result of the multiphoton image by comparing the multiphoton image with the H\&E-stained digital image.

2.4. Statistical Analysis. To further describe the differences between cytological features and collagen features in normal breast tissue, $\mathrm{ADH}$, low-grade DCIS, and high-grade DCISMI, three features were quantified from MPM images. The nuclear area of the epithelial cells within the ducts was used to describe differences in cytological features within the ducts, and collagen density was used to describe differences in collagen content around the ducts, and the basement membrane circumference was used to describe the extent of lesion involvement in the ducts. All statistical analyses were done using IBM SPSS Statistics 21. Statistical significance between each group was determined using the one-way ANOVA and was considered statistically significant when the $P$ value was $<0.05$.

\section{Result}

3.1. Multiphoton Microscopic Imaging. To demonstrate whether MPM has the ability to reveal differences in microstructure between normal breast tissue, ADH, DCIS, and DCIS-MI, MPM images of different samples were obtained and compared with corresponding H\&E images. During the multiphoton imaging scan of label-free breast tissue, a "screen door effect" appeared on the large-field multiphoton images (e.g., Figures 1(a), 1(b), 1(c), 1(i), 1(j), and 1(k)) causing a "grid" to appear on these images. However, when we divided a large-field-of-view image into four equal parts or eight equal parts for multiphoton imaging, the "screen door effect" was weakened significantly or does not appear on the multiphoton image. Figure 1 shows the representative MPM images and corresponding $\mathrm{H} \& \mathrm{E}$ images of normal breast tissue, ADH, DCIS, and DCIS-MI. Figure 2 shows representative MPM images and corresponding H\&E images of the microstructure of normal breast tissue, $\mathrm{ADH}$, low-grade DCIS, and high-grade DCIS-MI. As shown in Figure 1(c), 
TPER

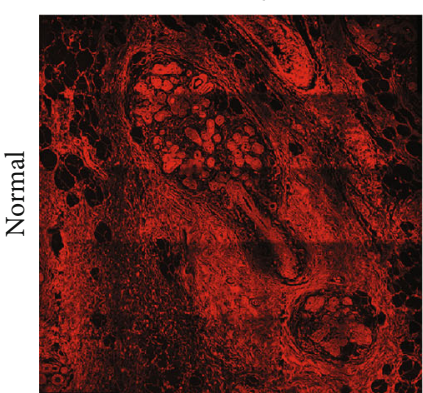

(a)

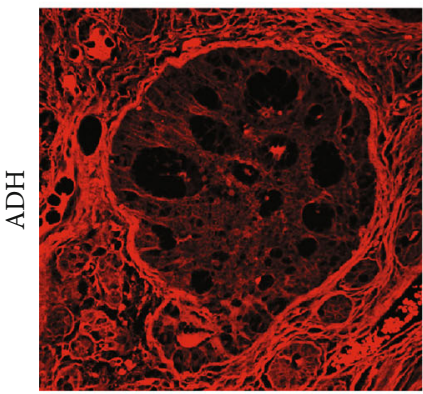

(e)

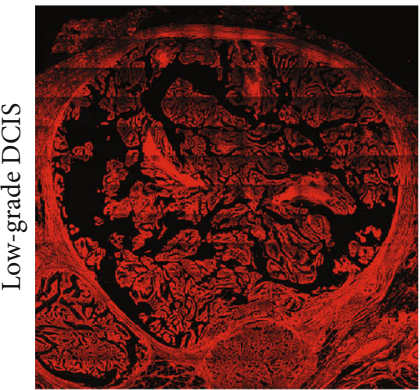

(i)

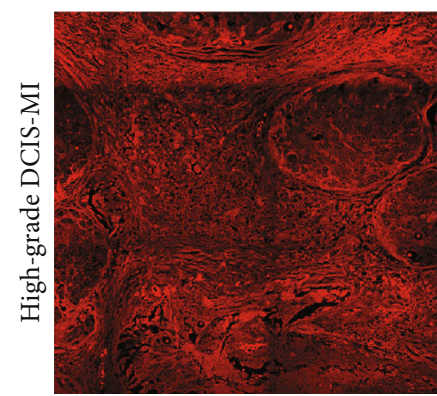

(m)

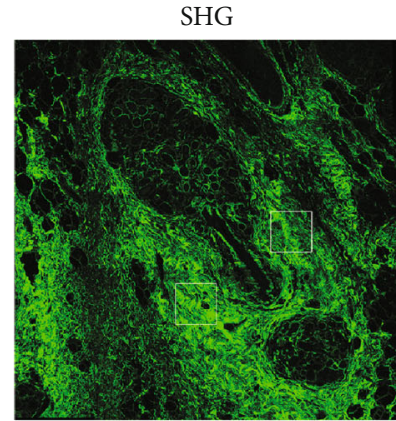

(b)

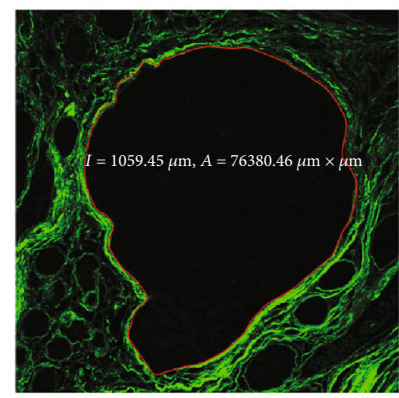

(f)

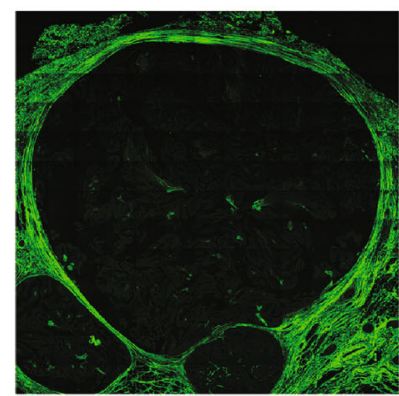

(j)

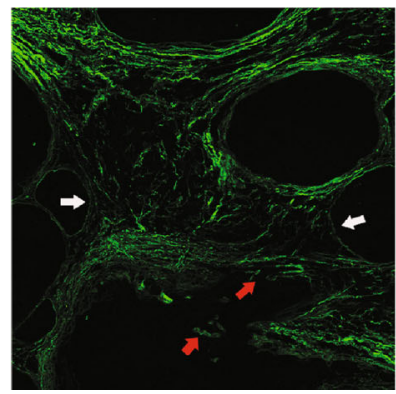

(n)

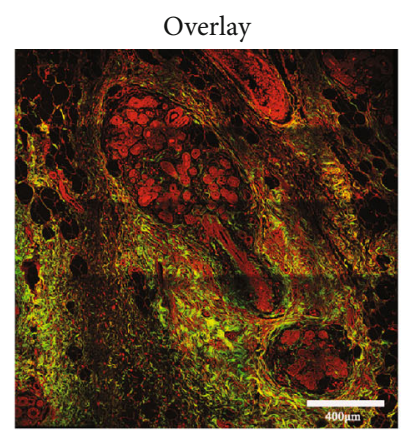

(c)

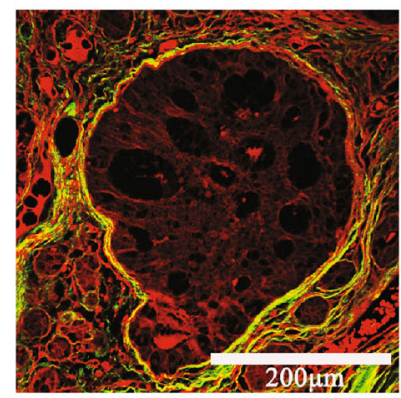

(g)

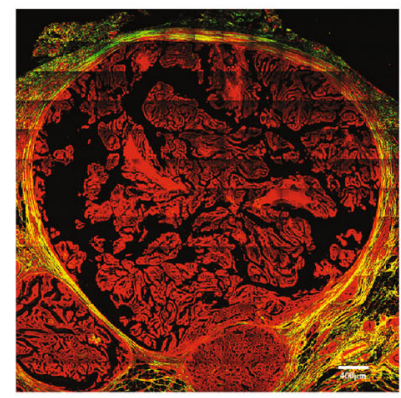

(k)

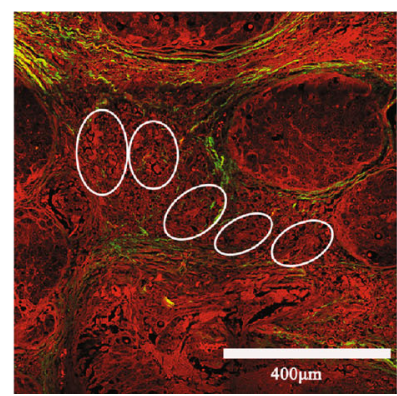

(o)

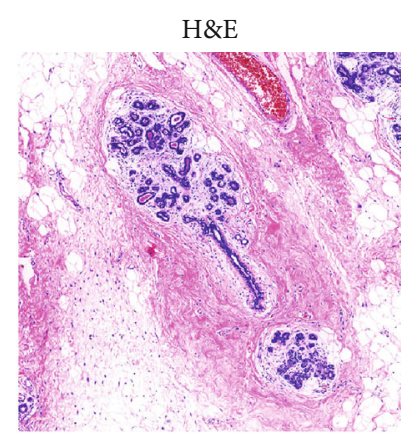

(d)

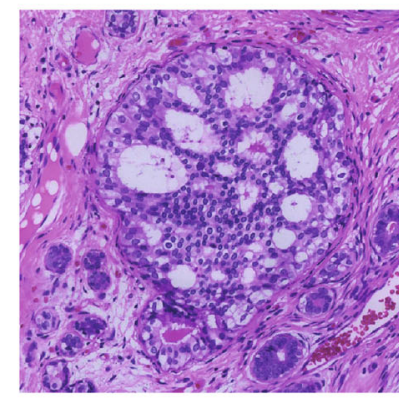

(h)

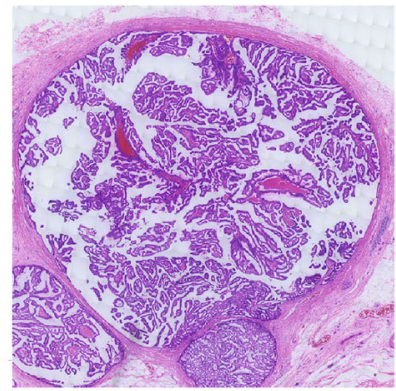

(l)

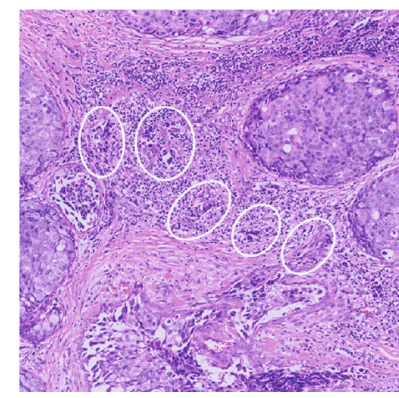

(p)

FIGURE 1: Representative MPM images and corresponding H\&E images of normal breast tissue, ADH, low-grade DCIS, and high-grade DCIS-MI. White arrow: complete basement membrane; red arrow: broken basement membrane; white oval: microinvasion cancer cells.

normal breast tissue consisted of two parts: one was the ductal-lobular system and the other was the stromal system composed of fibrous tissue and adipose tissue. According to previous reports and current experimental results, cells and elastic fibers are capable of producing TPEF signals (red color-coded) in Figure 1(a), whereas collagen in the extracellular matrix produces SHG (red color-coded) in Figure 1(b) [1]. Specifically, Figure 2(a) showed that the normal ductal was bilayered: the inner cell layer consists of epithelial cells, while the outer layer contains myoepithelial cells. Collagen fibers within stromal tissue were curved and dense, and basement membranes of the acinus and duct can be seen in Figure 1(b). The cytological features and positional alignment information of these cells in the MPM images were consistent with the corresponding H\&E images in Figures 1(d) and 2(e), whereas the H\&E images would not directly observe the distribution of collagen and the presence of the basement membrane.

Unlike the normal duct, $\mathrm{ADH}$ was a small-scale proliferation of epithelial cells within the duct, and these epithelial 


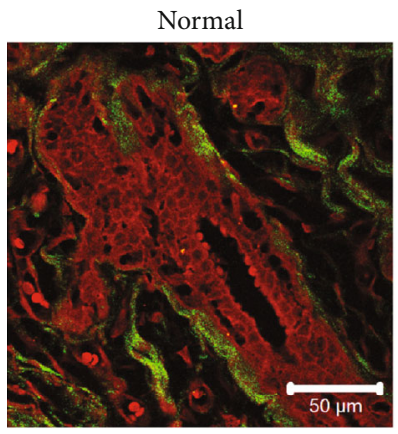

(a)

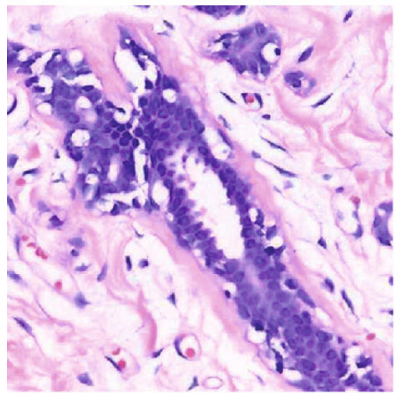

(e)

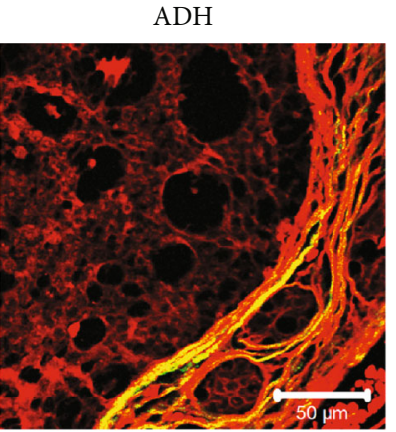

(b)

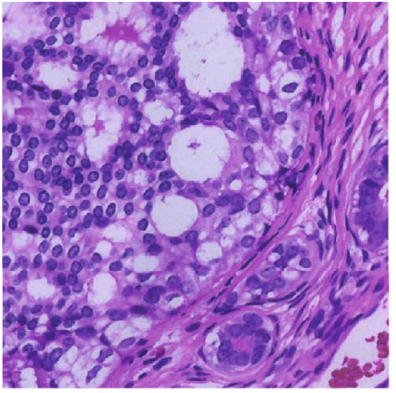

(f)

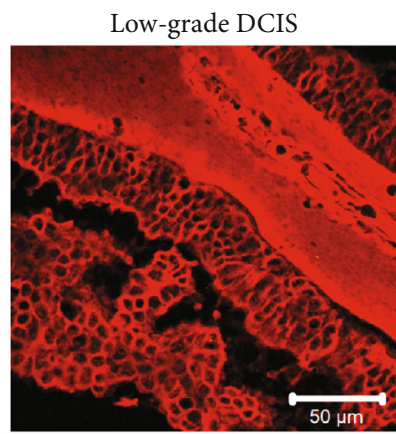

(c)

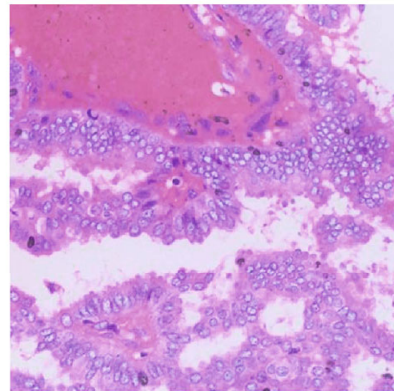

(g)

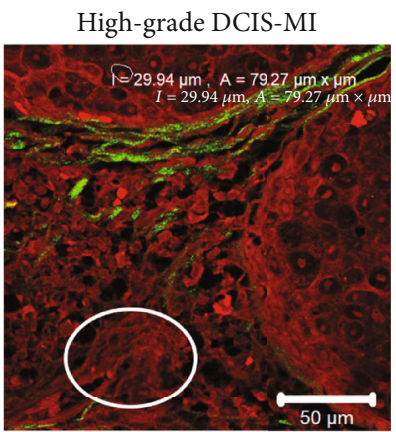

(d)

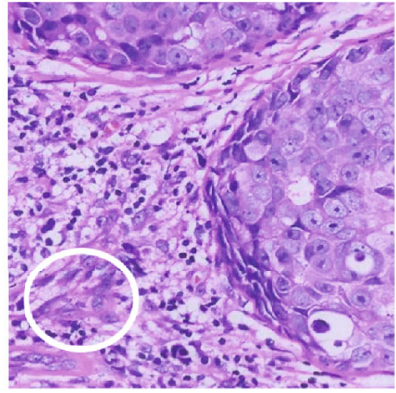

(h)

FIGURE 2: Representative MPM images and corresponding H\&E images of the microstructure of normal breast tissue, ADH, low-grade DCIS, and high-grade DCIS-MI. White oval: microinvasion cancer cells; scale bar: $50 \mu \mathrm{m}$.

cells were characterized by small uniform nuclei, as shown in the TPEF image (Figure 1(e)). As can be seen in Figure 1(f), the basement membrane of ADH excited strong SHG signals so that the basement membrane was clearly visible. Additionally, the MPM images (Figures $1(\mathrm{e})-1(\mathrm{~g})$ ) showed that the basement membrane was intact but significantly enlarged. The high-contrast overlay images (Figures $1(\mathrm{~g})$ and 2(b)) clearly showed the distribution and microstructure of collagen fibers and proliferating epithelial cells. These morphological details correlate well with the results of H\&E images (Figures 1(h) and 2(f)). However, H\&E images failed to show the microstructure and distribution of collagen fibers directly.

Figures 1(i)-1(l) show an example of MPM images and a corresponding H\&E image of low-grade DCIS. Figure 2 shows that epithelial cells proliferating in low-grade DCIS ducts were similar to those proliferating in ADH ducts. Hyperplastic epithelial cell populations are characterized by small and uniform nuclei. Unlike $\mathrm{ADH}$, low-grade DCIS involved more ducts or involved a larger duct (Figures $1(\mathrm{~g})$ and $1(\mathrm{k})$ ). As shown in Figure 1(k), epithelial cells proliferating in ducts involved a large duct and adjacent ducts. The strong SHG signal showed that the collagen fiber bundles in the basement membrane and stromal tissue were clearly visible, and the collagen fibers became straight due to the expansion of the ducts. The MPM images (Figure 2(c)) of lowgrade DCIS showed details of the hyperplastic epithelial cells that showed exactly the same details as the H\&E image (Figure 2(g)). However, the SHG image (Figure 1(j)) showed the distribution of collagen in the stromal tissue, which could not be directly observed from the H\&E images.

Compared with the ADH and DCIS, DCIS-MI is based on DCIS with cancer cells breaking through the basement membrane of the duct into the stromal tissue, but the maximum diameter of the largest infiltrating lesion is $\leq 1 \mathrm{~mm}$. Figures $1(\mathrm{~m})-1(\mathrm{p})$ show MPM images of high-grade DCISMI and a corresponding H\&E-stained image of high-grade DCIS-MI. Unlike the proliferating cells of low-grade DCIS, the proliferating cells of high-grade DCIS had larger cell volume, larger nuclei, obvious nuclear abnormalities, and nucleoli (Figure 1(m)). The SHG image (Figure 1(n)) displayed that the collagen around the ducts was unevenly distributed, and some of the collagen around the ducts was very thin, while some of the collagen around the ducts was very dense. The basement membrane of one of the ducts was destroyed, the collagen in the stromal tissue was no longer distributed around the duct but disorderly arranged, and the collagen density was also reduced as shown in Figure 1(n). The cytological features exhibited by MPM images were identical to those shown by H\&E images (Figures $1(\mathrm{p})$ and $2(\mathrm{~h})$ ), but the H\&E images were not able to directly exhibit morphological changes in the collagen in the basement membrane and stromal tissue.

Comparing MPM images of normal breast tissue and early stages of breast ductal carcinoma, including $\mathrm{ADH}$, low-grade DCIS, and high-grade DCIS-MI, MPM-identifiable features are summarized and shown in Table 1. MPM had the ability to identify early stages of breast ductal carcinoma by combining TPEF signals from cells and SHG signals from collagen in the stromal tissue. It revealed that MPM had the ability to provide accurate pathology information for clinical diagnosis.

3.2. Quantitative Analysis. In Figure 2, the TPEF signal intensity varies greatly between the nucleus and the cytoplasm, so we can manually draw the outline of the nucleus. As shown 
TABLE 1: MPM identifiable features of normal breast tissue, ADH, low-grade DCIS, and high-grade DCIS-MI.

\begin{tabular}{|c|c|c|}
\hline Breast tissue sample & TPEF signal & SHG signal \\
\hline Normal & Two layers of epithelial cells line in the duct & $\begin{array}{l}\text { Curly and rich collagen fibers in the stromal } \\
\text { Basement membrane outline is clearly visible } \\
\text { and complete }\end{array}$ \\
\hline $\mathrm{ADH}$ & $\begin{array}{l}\text { Small-scale epithelial cells proliferate in the duct } \\
\text { Complete involvement of fewer than } 2 \text { ducts } \\
\text { or }<2 \mathrm{~mm} \text { in extent }\end{array}$ & \multirow{2}{*}{$\begin{array}{c}\text { The basement membrane remains intact but } \\
\text { significantly enlarged } \\
\text { Collagen around the basement membrane becomes } \\
\text { fine as the duct expands } \\
\text { No significant change in the morphology of the } \\
\text { collagen in the stromal, but the collagen density } \\
\text { is reduced }\end{array}$} \\
\hline Low-grade DCIS & $\begin{array}{l}\text { Small, uniform cells with generally rounded nuclei } \\
\text { that are evenly spaced cells, proliferate in the ducts } \\
\text { A large number of proliferating cells involve more } \\
\text { than two ducts or involve large ducts }>2 \mathrm{~mm} \text { in diameter }\end{array}$ & \\
\hline $\begin{array}{l}\text { High-grade } \\
\text { DCIS-MI }\end{array}$ & $\begin{array}{l}\text { Large cells with distinct nucleoli, significant } \\
\text { nuclear abnormalities, proliferate in the duct } \\
\text { and stromal } \\
\text { A large amount of lymphocyte infiltration and a small } \\
\text { amount of malignant cell infiltrate in the stroma }\end{array}$ & $\begin{array}{c}\text { Some basement membranes remain intact, but } \\
\text { some basement membranes are no longer intact } \\
\text { The collagen bundles in the stromal become } \\
\text { straight and sparse }\end{array}$ \\
\hline
\end{tabular}

in Figure 2(d), we automatically got the nucleus area after manually drawing the outline of the nucleus in the ZEN back software. The nucleus was circled out manually on the ZEN back software to measure the size of the nucleus. Twenty well-defined nuclei were selected randomly from each normal, AHD, low-grade DCIS, and high-grade DCIS-MI sample. Specifically, the nuclear area was $16.12 \pm 3.10 \mu \mathrm{m}^{2}$ in normal breast tissue, $25.75 \pm 5.62 \mu \mathrm{m}^{2}$ in $\mathrm{ADH}, 27.10 \pm$ $5.28 \mu \mathrm{m}^{2}$ in low-grade DCIS, and $82.25 \pm 22.76 \mu \mathrm{m}^{2}$ in high-grade DCIS-MI. According to the one-way ANOVA test, we provided a bar chart (Figure 3(a)) of the statistics to show the significance between the different groups. Our results showed no statistical nuclear area difference $(P>0.05)$ between normal breast tissue and $\mathrm{ADH}(P=0.07)$ and between $\mathrm{ADH}$ and low-grade DCIS $(P=0.572)$. However, there were statistically significant differences between the other two groups $(P<0.05)$.

For each sample, three random SHG images of the same size $(512 \times 512$ pixels $)$ were selected to calculate the collagen density. Collagen density is defined as the ratio of the pixel number $\mathrm{A}$ to the total of $\mathrm{B}$, and the pixel number $\mathrm{A}$ is the pixel number of the total pixel number $B$ minus the low threshold number (low-intensity SHG signal and background signal). To avoid the influence of the "grid" on the quantitative measurement of collagen density, we chose the measurement position (ROI) in the middle of each "grid (size $1024 \times 1024$ pixels)"; for example, we selected the position and size of the white rectangle in Figure 1(b) to quantify the collagen density. The mean and SD of the collagen density were $0.86 \pm 0.06$ for normal breast tissue, $0.67 \pm 0.09$ for $\mathrm{ADH}, 0.57 \pm 0.137$ for low-grade DCIS, and $0.42 \pm$ 0.162 for high-grade DCIS-MI. The bar chart (Figure 3(b)) of the statistics showed statistically significant difference $(P<0.05)$ in collagen density between any two groups.

To quantify the size of normal ducts and the size of lesions involved, we used the ZEN back software to manually circle the basement membrane and obtain automatically its circumference, as shown in Figure 1(f). To reduce the influ- ence of the "grid" phenomenon on the quantitative measurement of the basement membrane circumference, we chose the duct basement membrane in the image without the "grid" for measurement, except for Figure 1(j). For normal and low-grade DCIS samples, three complete ducts were selected for each sample to calculate the basement membrane circumference. However, for $\mathrm{ADH}$ samples, one or two complete ducts were selected for each sample to calculate the basement membrane circumference. To be specific, the basement membrane circumference in normal ducts was $149.32 \pm 12.56 \mu \mathrm{m}$, in ADH ducts was 958.96 $\pm 101.63 \mu \mathrm{m}$, and in low-grade DCIS was $2474.35 \pm 1050.11 \mu \mathrm{m}$, whereas, in high-grade DCIS-MI, it is " $\infty$ " because the high-grade DCIS-MI has some incomplete basement membranes. We did the one-way ANOVA test of the basement membrane circumference and found that there was no statistically significant difference $(P>0.05)$ between the normal case and ADH $(P=0.41)$, and the other two groups showed statistically significant difference $(P<0.05)$, as shown in Figure $3(c)$.

\section{Discussion}

MPM, targeting SHG and TPEF, has been widely used as a very useful tool in cancer research and detection, such as intraoperative real-time detection, marginal implementation assessment, and cancer diagnosis [13, 19, 21-24]. Moreover, MPM also plays an important role in breast cancer research, including rapid intraoperative evaluation of breast lesions, detection of breast tumor progression, and the role of collagen density in the mammary tumor initiation and progression $[15,25,26]$. However, few MPM studies have been conducted on these early stages of breast ductal carcinoma [16], especially on MPM imaging of ADH and DCIS-MI. In recent years, these early stages of breast ductal carcinoma have attracted more and more scholars' attention because of the good prognosis of these lesions after treatment [27]. The identification of DCIS and DCIS-MI is a difficult point in the breast pathological diagnosis. The presence of cancer 


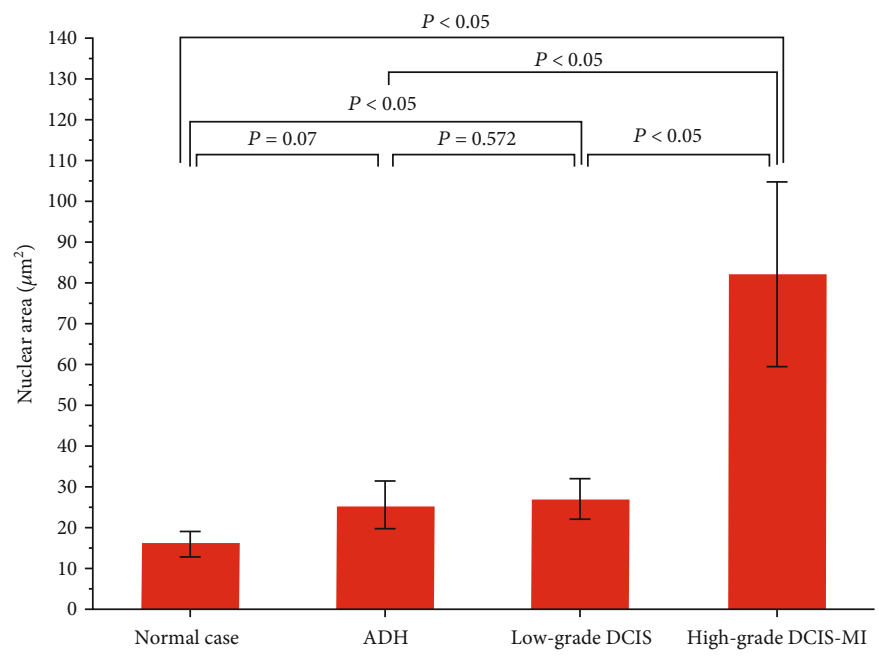

(a)

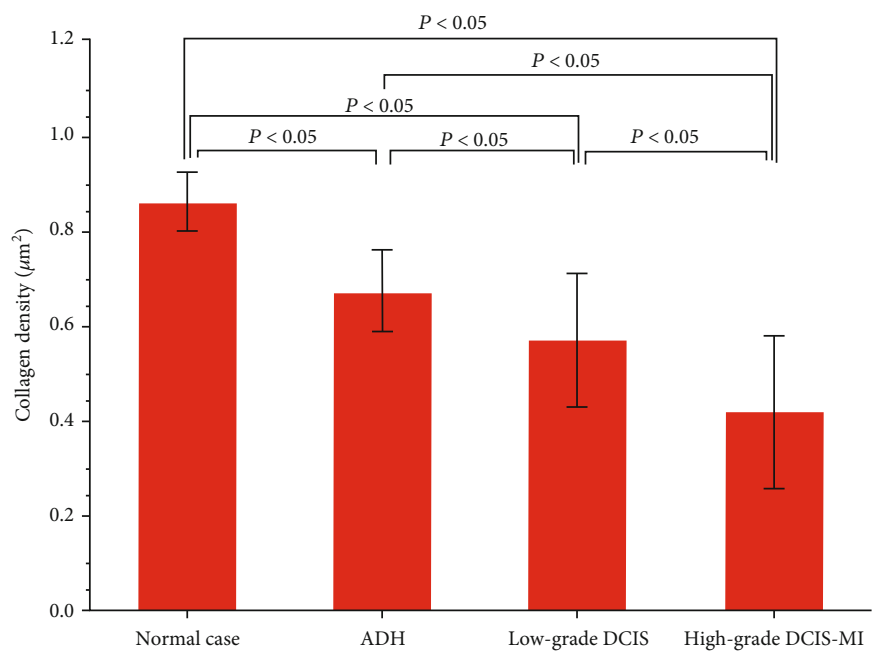

(b)

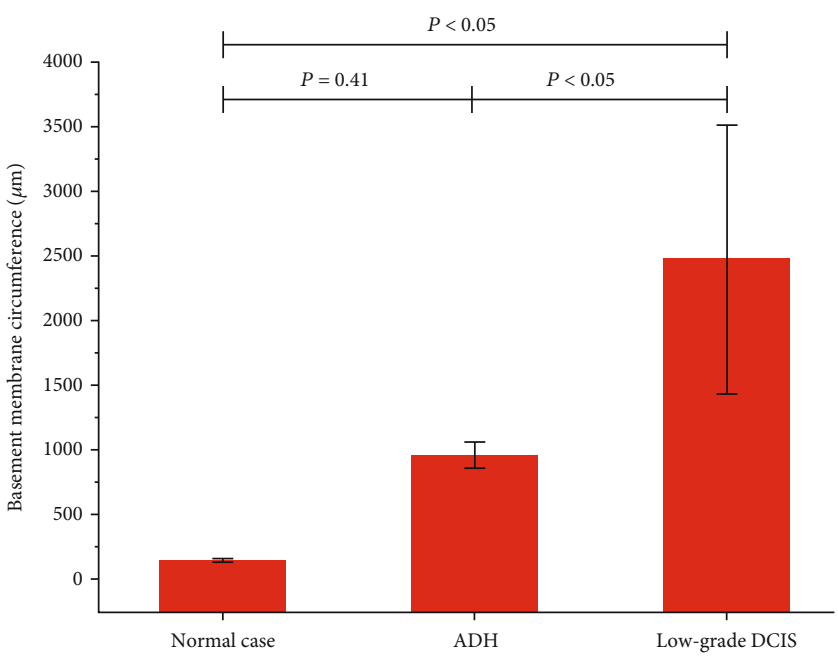

(c)

FIGURE 3: Nuclear area (a) and collagen density (b) of normal breast tissue versus ADH and low-grade DCIS as well as high-grade DCIS-MI; the basement membrane circumference (c) of normal breast tissue versus ADH and low-grade DCIS; error bars: standard deviation.

cell infiltrates is a central concern for clinicians and pathologists because of the different treatment strategies for DCIS and DCIS-MI.

In the early-stage lesions of breast ductal carcinoma, there must be differences in cytological features, which are a very important indicator of tumor staging [25]. The nucleus of high-grade DCIS-MI was significantly larger than that of DCIS and ADH, as shown in Figures 2 and 3(a). The nuclei of normal breast tissue, $\mathrm{ADH}$, and low-grade DCIS were mostly oval. However, the nuclei of high-grade DCIS-MI vary widely in size, with round and oval shapes. Additionally, breast lesions also have different changes in the extracellular matrix at different stages. We did not find any statistically significant differences in the nuclear area and basement membrane circumference between normal breast tissue and $\mathrm{ADH}$ (Figures 3(a) and 3(c)). But we found statistically significant differences in collagen density in the stromal tissue between normal breast tissue and $\mathrm{ADH}$. Therefore, the ability to visualize cytological features and collagen morphology in the extracellular matrix is very helpful in identifying early stages of breast ductal carcinoma. In this study, we used MPM to detect cytological features and collagen structure characteristics by combining TPEF signals from intracellular autofluorescence and SHG signals from collagen in the extracellular matrix [28].

Our results reveal that MPM is an efficient and promising clinical tool for identifying early stages of breast ductal carcinoma. It not only provides high-resolution cytological features comparable to H\&E-stained slice images of the "gold standard" of pathology but also provides additional collagen imaging information for extracellular matrices [22]. Quantitative results of nuclear area and basement membrane circumference revealed that $\mathrm{ADH}$ and low-grade DCIS have similar cytological features, but low-grade DCIS had a wider range of lesions, and involve more ducts. Compared with $\mathrm{ADH}$ and low-grade DCIS, high-grade DCIS-MI has larger nuclei, and some of the ductal basement membranes were destroyed, causing some cancer cells to propagate into 
adjacent stromal tissue. The quantitative statistical results of collagen density reflect changes of the extracellular matrix collagen content, which is an important indicator of tumor staging [25]. Therefore, these three variable analyses would enable identifying the early stages of breast ductal carcinoma.

Compared to fresh unfixed tissue samples, MPM imaging of paraffin section samples has a strong fluorescent background due to formalin fixation [29, 30]. Therefore, the image background is more uniform and can produce good image contrast with the nucleus or other components. In this study, TPEF signals were mainly generated from elastic fibers, $\mathrm{NAD}(\mathrm{P}) \mathrm{H}, \mathrm{FAD}$, and formalin solutions. The TPEF signal in the fixed high-grade nucleus mainly comes from the formalin solution and does not change with the change of fixed conditions. In Figures 1(o) and 2(d), we observed that there were one or more red dots (nucleoli) in the highgrade nuclei, but there was no TPEF signal (red dots) in the high-grade nuclei in fresh unfixed breast samples [25]. On the other hand, MPM imaging of fresh unfixed tissue samples can reduce the time for rapid intraoperative diagnosis because fresh samples do not require steps such as fixation and staining. However, in actual clinical procedures, fresh tissue samples of ADH and DCIS-MI are more difficult to collect.

With the increasing attention on MPM, many studies have demonstrated that MPM has great advantages in the feasibility of clinical translation [20, 25, 31, 32]. However, compared to currently accepted clinical imaging systems (ultrasound, X-ray computed tomography or tomosynthesis, and MRI), MPM has limited penetration depth for highresolution imaging of tissues. Miniature multiphoton endoscopes and flexible small-diameter MPM probes overcome the previous limitations of this technology [33-36]. Huland et al. and Demirhan et al. have developed a compact and flexible multiphoton microendoscope to observe the liver, kidney, and colon of anesthetized rats $[33,34]$. Yan et al. used a fluorescence endoscopy imaging system based on gradient index (GRIN) lenses to achieve in vivo dynamic fluorescence microendoscopic imaging and monitored the movement of subcutaneous blood flow in anesthetized mice [35]. In addition, Karsten et al. successfully exceeded the $600 \mathrm{~Hz}$ frame rate with a multifocal multiphoton endoscope [37]. Therefore, our studies on ex vivo human breast tissue will provide the groundwork for further application of MPM in vivo and in clinic, such as real-time diagnosis of intraoperative tissue and postoperative pathological analysis.

\section{Conclusion}

In conclusion, MPM is a very useful tool for label-free identification of early stages of breast ductal carcinoma, including $\mathrm{ADH}$, low-grade DCIS, and high-grade DCISMI. Through high-contrast MPM images, we can analyze quantitatively the microstructural changes in the nucleus, basement membrane, and collagen density to distinguish the early stages of breast ductal carcinoma. Multiphoton microscopy via TPEF combined with SHG has the potential to be used for clinical diagnosis and lead to better management of these diseases.

\section{Data Availability}

All data needed to evaluate the conclusions in the paper are present in the paper. Additional data related to this paper may be requested from the authors.

\section{Conflicts of Interest}

The authors declare no conflict of interests.

\section{Authors' Contributions}

Zhong Chen, Wenhui Guo, and Deyong Kang contributed equally to this work.

\section{Acknowledgments}

This work was supported by the National Natural Science Foundation of China (Grant No. 81671730), the Joint Funds of Fujian Provincial Health and Education Research (WKJ2016-2-28), the Special Funds of the Central Government Guiding Local Science and Technology Development (2017L3009), and the Program for Chang Jiang Scholars and Innovative Research Team in University (IRT_15R10).

\section{References}

[1] R. L. Siegel, K. D. Miller, and A. Jemal, "Cancer statistics, 2018," CA: A Cancer Journal for Clinicians, vol. 68, no. 1, p. 11, 2018.

[2] R. Vajpeyi, "WHO classification of tumours: pathology and genetics of tumours of the breast and female genital organs," Journal of Clinical Pathology, vol. 76, no. 1, pp. 139-141, 2005.

[3] S. R. Wellings, H. M. Jensen, and R. G. Marcum, "An atlas of subgross pathology of the human breast with special reference to possible precancerous lesions," Journal of the National Cancer Institute, vol. 55, no. 2, pp. 231-273, 1975.

[4] L. C. Hartmann, D. C. Radisky, M. H. Frost et al., "Understanding the premalignant potential of atypical hyperplasia through its natural history: a longitudinal cohort study," Cancer Prevention Research, vol. 7, no. 2, pp. 211-217, 2014.

[5] S. R. Wellings and H. M. Jensen, "On the origin and progression of ductal carcinoma in the human breast," Journal of the National Cancer Institute, vol. 50, no. 5, pp. 1111-1118, 1973.

[6] M. A. Lopez-Garcia, F. C. Geyer, M. Lacroix-Triki, C. Marchió, and J. S. Reis-Filho, "Breast cancer precursors revisited: molecular features and progression pathways," Histopathology, vol. 57, no. 2, pp. 171-192, 2010.

[7] I. de Mascarel, G. MacGrogan, S. Mathoulin-Pélissier, I. Soubeyran, V. Picot, and J.-M. Coindre, "Breast ductal carcinoma in situ with microinvasion," Cancer, vol. 94, no. 8, pp. 2134-2142, 2010.

[8] AJCC, Cancer Staging Manual, Springer, New York, NY, USA, 8th edition, 2017.

[9] D. C. Allred, "Ductal carcinoma in situ: terminology, classification, and natural history," Journal of the National Cancer Institute Monographs, vol. 2010, no. 41, pp. 134-138, 2010.

[10] D. L. Page, W. D. Dupont, L. W. Rogers, and M. S. Rados, "Atypical hyperplastic lesions of the female breast. A longterm follow-up study," Cancer, vol. 55, no. 11, pp. 2698-2708, 2015. 
[11] F. A. Tavassoli and H. J. Norris, "A comparison of the results of long-term follow-up for atypical intraductal hyperplasia and intraductal hyperplasia of the breast," Cancer, vol. 65, no. 3, pp. 518-529, 1990.

[12] I. Pavlova, K. R. Hume, S. A. Yazinski, R. M. Peters, R. S. Weiss, and W. W. Webb, "Multiphoton microscopy as a diagnostic imaging modality for lung cancer," in Proceedings Volume 7569, Multiphoton Microscopy in the Biomedical Sciences $X$, San Francisco, CA, USA, Febuary 2010.

[13] P. P. Provenzano, K. W. Eliceiri, J. M. Campbell, D. R. Inman, J. G. White, and P. J. Keely, "Collagen reorganization at the tumor-stromal interface facilitates local invasion," BMC Medicine, vol. 4, no. 1, p. 38, 2006.

[14] P. P. Provenzano, D. R. Inman, K. W. Eliceiri et al., "Collagen density promotes mammary tumor initiation and progression," BMC Medicine, vol. 6, no. 1, p. 11, 2008.

[15] M. Sidani, J. Wyckoff, C. Xue, J. E. Segall, and J. Condeelis, "Probing the microenvironment of mammary tumors using multiphoton microscopy," Journal of Mammary Gland Biology and Neoplasia, vol. 11, no. 2, pp. 151-163, 2006.

[16] Y. Wu, F. Fu, Y. Lian, Y. Nie, C. Wang, and J. X. Chen, "Identifying three different architectural subtypes of mammary ductal carcinoma in situ using multiphoton microscopy," Journal of Physics D: Applied Physics, vol. 48, no. 40, article 405401, 2015.

[17] W. R. Zipfel, R. M. Williams, R. Christie, A. Y. Nikitin, B. T. Hyman, and W. W. Webb, "Live tissue intrinsic emission microscopy using multiphoton-excited native fluorescence and second harmonic generation," Proceedings of the National Academy of Sciences of the United States of America, vol. 100, no. 12, pp. 7075-7080, 2003.

[18] V. Lutz, M. Sattler, S. Gallinat, H. Wenck, R. Poertner, and F. Fischer, "Characterization of fibrillar collagen types using multi-dimensional multiphoton laser scanning microscopy," International Journal of Cosmetic Science, vol. 34, no. 2, pp. 209-215, 2003.

[19] K. Burke, M. Smid, R. P. Dawes et al., "Using second harmonic generation to predict patient outcome in solid tumors," BMC Cancer, vol. 15, no. 1, pp. 1-10, 2015.

[20] S. Wang, X. Wang, J. Chen et al., "Optical visualization of cerebral cortex by label-free multiphoton microscopy," IEEE Journal of Selected Topics in Quantum Electronics, vol. 25, no. 1, pp. 1-8, 2019.

[21] Y. Sun, S. You, H. Tu et al., "Intraoperative visualization of the tumor microenvironment and quantification of extracellular vesicles by label-free nonlinear imaging," Science Advances, vol. 4 , no. $12,2018$.

[22] P. P. Provenzano, C. T. Rueden, S. M. Trier et al., "Nonlinear optical imaging and spectral-lifetime computational analysis of endogenous and exogenous fluorophores in breast cancer," Journal of Biomedical Optics, vol. 13, no. 3, article 031220, 2008.

[23] U. Aparna Dravid and N. Mazumder, "Types of advanced optical microscopy techniques for breast cancer research: a review," Lasers in Medical Science, vol. 33, no. 9, pp. 18491858, 2018.

[24] S. W. Perry, R. M. Burke, and E. B. Brown, "Two-photon and second harmonic microscopy in clinical and translational cancer research," Annals of Biomedical Engineering, vol. 40, no. 2, pp. 277-291, 2012.

[25] Y. Wu, F. Fu, Y. Lian et al., "Monitoring morphological alterations during invasive ductal breast carcinoma progression using multiphoton microscopy," Lasers in Medical Science, vol. 30, no. 3, pp. 1109-1115, 2015.

[26] Y. K. Tao, D. Shen, Y. Sheikine et al., "Assessment of breast pathologies using nonlinear microscopy," Proceedings of the National Academy of Sciences of the United States of America, vol. 111, no. 43, pp. 15304-15309, 2014.

[27] K. E. Stuart, N. Houssami, R. Taylor, A. Hayen, and J. Boyages, "Long-term outcomes of ductal carcinoma in situ of the breast: a systematic review, meta-analysis and meta-regression analysis," BMC Cancer, vol. 15, no. 1, p. 890, 2015.

[28] L. Li, D. Kang, C. Feng et al., "Label-free assessment of premalignant gastric lesions using multimodal nonlinear optical microscopy," IEEE Journal of Selected Topics in Quantum Electronics, vol. 25, no. 1, pp. 1-6, 2019.

[29] W. Beisker, F. Dolbeare, and J. W. Gray, "An improved immunocytochemical procedure for high-sensitivity detection of incorporated bromodeoxyuridine," Cytometry Part A, vol. 8, no. 2, pp. 235-239, 2010.

[30] N. Billinton and A. W. Knight, "Seeing the wood through the trees: a review of techniques for distinguishing green fluorescent protein from endogenous autofluorescence," Analytical Biochemistry, vol. 291, no. 2, pp. 175-197, 2001.

[31] W. R. Zipfel, R. M. Williams, and W. W. Webb, "Nonlinear magic: multiphoton microscopy in the biosciences," Nature Biotechnology, vol. 21, no. 11, pp. 1369-1377, 2003.

[32] M. J. Huttunen, A. Hassan, C. W. Mccloskey et al., "Automated classification of multiphoton microscopy images of ovarian tissue using deep learning," Journal of Biomedical Optics, vol. 23, no. 6, pp. 1-7, 2018.

[33] D. M. Huland, C. M. Brown, S. S. Howard et al., "In vivo imaging of unstained tissues using long gradient index lens multiphoton endoscopic systems," Biomedical Optics Express, vol. 3, no. 5, pp. 1077-1085, 2012.

[34] D. Kobat, N. G. Horton, and C. Xu, "In vivo two-photon microscopy to 1.6-mm depth in mouse cortex," Journal of Biomedical Optics, vol. 16, no. 10, article 106014, 2011.

[35] W. Yan, X. Peng, D. Lin et al., "Fluorescence microendoscopy imaging based on GRIN lenses with one- and two-photon excitation modes," Frontiers of Optoelectronics, vol. 8, no. 2, pp. 177-182, 2015.

[36] M. E. Bocarsly, W.-c. Jiang, C. Wang, J. T. Dudman, N. Ji, and Y. Aponte, "Minimally invasive microendoscopy system for in vivo functional imaging of deep nuclei in the mouse brain," Biomedical Optics Express, vol. 6, no. 11, pp. 4546-4556, 2015.

[37] K. Bahlmann, P. T. So, M. Kirber et al., "Multifocal multiphoton microscopy (MMM) at a frame rate beyond $600 \mathrm{~Hz}$," Optics Express, vol. 15, no. 17, pp. 10991-10998, 2007. 